\title{
Sentimientos, afectos y lógica afectiva. Su lugar en nuestra comprensión del otro y del mundo
}

El peso de los sentimientos, las emociones o los afectos en nuestra comprensión del prójimo y del mundo es un tema eludido conscientemente, durante mucho tiempo, por la ciencia y los científicos. Y esto se explica por las numerosas contradicciones e inexactitudes que, desde siempre, han enturbiado el problema de tales sentimientos. Sin embargo, es cierto que todos nosotros somos, de alguna manera, «expertos en sentimientos»e, incluso, también es verdad que vivimos toda nuestra vida, como bien decía Hermann Hesse, esencialmente «a través de los sentimientos» ${ }^{1}$. Aunque, por otro lado, no sabemos, o al menos la ciencia no lo sabe con certeza, qué es un sentimiento, cuál es su significado y cómo actúa. Teniendo en cuenta que de manera habitual los sentimientos se consideran inasibles, irracionales y perturbadores, admitimos que deben eliminarse en lo posible de cualquier «pensamiento objetivo». De ahí deducimos que cualquier ánimo de comprender al otro o al mundo se nos presenta tergiversado y exclusivamente cerebral, lo que tiene profundas implicaciones no sólo respecto a nuestra actitud frente al mundo en general sino también frente a nuestro enfoque terapéutico.

Por otro lado, resulta muy sorprendente que las emociones jueguen un papel tan importante en cualquier acontecimiento psíquico o social de relieve -pensemos por un momento en los estallidos de violencia, en las revoluciones y en las guerras o, en general, en todo lo que ocurre a diario en el ámbito familiar, profesional o político, y que, sin embargo, no ocupan el lugar que mínimamente les correspondería en cualquier teoría psicológica o sociológica, y tampoco que yo sepa, en ninguna teoría antropológica, evolucionista o cognitiva. Incluso en la ya centenaria teoría psicoanalítica-cuya dinámica de los sentimientos constituía desde sus inicios un foco de interés primordial- la noción de afecto ${ }^{2}$ aparece sorprendentemente como algo confuso y contradictorio, según Otto Kernberg, uno de los más eminentes teóricos psicoanalistas ${ }^{3}$. En cualquier contexto se hace necesaria una teoría clara y consistente, que incluya también las recientes investigaciones sobre neurobiología y psicología emocional. De hecho, siempre se echa en falta un lenguaje y una conceptualización científica comunes que permitan agru-

1 «Womit leben wir denn eigentlich, wo spüren wir das Leben, wenn nicht mit unserem Gefühl?», Hesse, H., 1927, p. 43.

2 «Affekt» en alemán, «affect» en inglés.

3 Kernberg, 1990. 
par de modo coherente, el cúmulo de nociones parciales y dispersas sobre las emociones que en tantos dominios aparecen.

Incluso el último replanteamiento de la postura casi siempre negativa hacia los sentimientos no cambia las cosas. Sabemos que ya la generación del sesenta y ocho exigía vivir mucho más «visceralmente». Durante cierto tiempo, un sector de las feministas defendía ideas muy parecidas argumentando -insostenible en este caso- que los sentimientos únicamente pertenecían al ámbito de lo femenino. Desde hace más de veinte años, la ciencia igualmente $-\mathrm{y}$ ante todo la ciencia del cerebro- se interesa cada vez más por las emociones. En psicología por añadidura se habla de un nuevo «giro emocional» que estaría a punto de sustituir al «giro cognoscitivo» de los años sesenta, marcado por el abandono de un conductismo puro y duro. Libros para el gran público como La inteligencia emocional de David Goleman o El error de Descartes de Antonio Damasio, ambos procedentes de las neurociencias modernas, han contribuido a difundir la nueva era emocional.

Presento, ahora, algunas informaciones sobre mi propio trabajo en este campo. Frustrado, de un lado, por las lagunas mencionadas y, de otro, fascinado por las convergencias subyacentes que creía detectar tras la multiplicidad de datos provenientes de diversos campos de investigación, trabajo desde hace más de veinte años en la problemática de las interacciones entre sentimiento y pensamiento desde un punto de vista científico. Mi punto de partida ha sido las experiencias psiquiátricas y psicoterapéuticas así como las extensas investigaciones sobre la dinámica evolutiva de los distintos trastornos psíquicos, en particular, de la esquizofrenia.

En 1982 presenté mis ideas en un primer libro titulado La lógica afectiva ${ }^{4}$, un concepto que implica a la vez la presencia de componentes lógicos en cualquier tipo de afecto, y la presencia de componentes afectivos en cualquier tipo de lógica. En 1997 publiqué una nueva síntesis de mis estudios, Las bases emocionales del pensamiento $^{5}$, que traspasaba los límites de la psiquiatría. Las principales bases de este acercamiento sistémico de elementos clave de orden neurobiológico, psicodinámico y sociodinámico son, por una parte, los estudios fundamentales de Jean Piaget sobre la génesis de las estructuras psíquicas en el niño, y, por otra, los descubrimientos de Konrad Lorenz sobre las raíces evolutivas de la mente. Una gran parte de este segundo libro ha sido escrito en un ambiente interdisciplinario único en el Instituto Konrad Lorenz, en Altenberg, cerca de Viena, donde tuve el privilegio de trabajar durante varios meses como profesor invitado, tras mi retirada de la vida universitaria.

«Affektlogik» en alemán, cf. Ciompi, 1982.

Ciompi, 1997. 
A continuación voy a resumir en cinco tesis fundamentales las interacciones principales entre sentimiento y pensamiento, tal y como se presentan desde el prisma de la lógica afectiva. Más adelante, abordaré algunas de las consecuencias prácticas y generales que se derivan de ello. Se revelará esencialmente -como desde ahora deseo indicar- que los sentimientos, emociones o afectos (ya hablaremos sobre la definición) juegan en cualquier tipo de pensamiento y acción un papel que no sólo es muy importante sino que es mucho más asible de lo que se admite.

\section{Cinco tesis esenciales para la lógica afectiva}

Mi primera tesis, que coincide además con el punto de partida de cualquier lógica afectiva postula que: sentimiento y pensamiento, -o emoción y cognición, afectividad y lógica, en un sentido amplio-, interactúan obligatoriamente en la actividad psíquica.

En sí mismo no se trata de un concepto nuevo; se corresponde con nuestra experiencia subjetiva diaria, constituye igualmente un postulado central del psicoanálisis desde sus inicios, que no ha sido seriamente cuestionado, en principio, por ninguna de las teorías cognoscitivas o emocionales existentes, incluida la epistemología genética de Jean Piaget. Sin embargo, las afirmaciones más sustanciales provienen de la investigación neurobiológica moderna que demuestra claramente que los centros cerebrales emocionales y cognoscitivos están íntimamente relacionados y se influyen mutuamente de continuo. En las amígdalas -un centro de primera importancia para la regulación de las emociones- cualquier estímulo sensorial que entra en el cerebro recibe obligatoriamente una «coloración emocional» que depende del contexto y de la experiencia vivida. Esta «coloración» afectará después en cómo la información sensorial sea memorizada, reactivada y unida a otras nociones cognoscitivas. El alcance de estos datos todavía no ha sido, en mi opinión, suficientemente comprendido. Esto significa, ni más ni menos, que el pensamiento «puro»y exento de afecto no existe ni en la ciencia, ni en la lógica formal, ni siquiera en las matemáticas, como veremos mejor enseguida. El problema se deriva de las implicaciones de este hecho, y precisamente esta problemática, bastante nueva, es lo que se propone estudiar la lógica afectiva.

Antes de proseguir, es necesario superar una etapa intermedia cuya importancia no puede subestimarse, como puede verse a continuación. Se trata de esclarecer lo que significa «afecto». En la literatura científica términos parcialmente superponibles como afecto, emoción, sentimiento o humor se siguen utilizando de manera aleatoria. Así, Kleinginna y Kleinginna, una pareja de investigadores americanos, han evidenciado en la literatura especializada más de 92 definiciones sólo 
para el término «emoción» ${ }^{6}$ (sin olvidar que este término fue introducido en ciencia por Descartes). Tan equívoco es el concepto del afecto - pues se entiende como una noción general que agrupa casi todos los fenómenos emocionales posibles y también como una noción muy restrictiva que se limita a un pequeño subgrupo de fenómenos emocionales que se corresponden con pasar a actuar impulsiva e incontroladamente- que cualquiera que se dedique científicamente a este campo está obligado a seleccionar y aclarar sus definiciones.

En el ámbito de la teoría de la lógica afectiva, el término «afecto» se utiliza constantemente en su primer sentido, es decir, como una noción general que engloba todos los fenómenos emocionales. Partiendo de un denominador común que a mi juicio reúne estos fenómenos, un afecto puede definirse como un estado psicofísico global de calidad, duración y grado de consciencia variables. Un afecto, por lo tanto, puede ser consciente o -lo que es todavía más importante- muy inconsciente. Su duración puede variar entre segundos, minutos o incluso horas o días, o, hasta semanas (como por ejemplo en estados depresivos o maníacos patológicos). Cualquier afecto constituye, además, un fenómeno típicamente psicosomático; generalmente se manifiesta simultáneamente en lo psíquico y (a veces casi de manera exclusiva) en lo corporal. Un afecto hace «latir el corazón», «da escalofríos», «remueve las entrañas». Repercute mucho en la mímica, los gestos, la postura corporal, en resumen, en la psicomotricidad en general. Es importante darse cuenta de que nunca estamos libres de afecto, pues el organismo se halla siempre en un estado global que corresponde a la definición aquí señalada. Incluso la quietud, la tranquilidad, la «neutralidad» o la indiferencia representan, igualmente, estados afectivos en el sentido descrito; siempre tienen efectos significativos sobre todo tipo de pensamiento y de comportamiento.

Otra noción que conduce, como veremos más adelante, a consecuencias particularmente interesantes es el aspecto energético de los afectos. El afecto corresponde, a mi entender, a un estado energético dirigido de manera concreta $\mathrm{o}$, de manera más precisa, a una distribución específica de energía conectada en el transcurso de la evolución a ciertas percepciones y comportamientos de importancia vital. Precisemos, sin embargo (para evitar malentendidos) que la noción de energía no implica en este caso una misteriosa «energía vital» de carácter «sublime», sino que hablamos de energías biológicas ordinarias tales como las que se introducen en el organismo mediante la nutrición para después ser gastadas, dependiendo del estado emocional predominante. En los estados de rabia o de pánico que llamamos simpaticotónicos, por ejemplo, enormes cantidades de energía pueden movilizarse y derrocharse en un lapso de tiempo extremadamente corto, al

6 Kleinginna y otros, 1981. 
servicio de los esfuerzos de combate o de huida. En los estados parasimpaticotónicos, por el contrario, -que se caracterizan por sentimientos de júbilo, amor o sosiego al servicio de la relajación, ingestión de alimentos o de la vida social, incluida la sexualidad-, la energía biológica se ingiere o se derrocha de manera lenta y mesurada, dependiendo de la especificidad para cada efecto.

Solo digo que la ciencia está en estos momentos a punto de identificar, con precisión, un pequeño número de estados genéticamente determinantes, llamados afectos de base, como son la curiosidad o el interés, el miedo, el enfado, la alegría y la tristeza, y según ciertos autores, también el temor, el hastío y la vergüenza. Los innumerables matices que hacen que el mundo de las emociones sea tan difícilmente de delimitar se entienden bien como una mezcla, bien como una modulación cultural de afectos de base. De manera todavía más radical, la multitud de afectos puede también reducirse a la única dicotomía entre sentimientos positivos y negativos, es decir, entre placer y displacer. A diferencia de los prejuicios comunes, el mundo de los afectos no es forzosamente más complejo sino en realidad más simple que el mundo potencialmente infinito de la cognición, en lo que respecta a la percepción y al pensamiento.

El concepto de cognición dista mucho de utilizarse unívocamente en la literatura científica. Bajo la influencia de la espectacular expansión de las «neurociencias cognitivas», se extiende también hacia el campo de las emociones. Con todo, la primera condición para una exploración fructífera de las interacciones entre emoción y cognición consiste en una precisa delimitación entre ambas nociones. Por lo tanto, el concepto de cognición se define, en el marco de la lógica afectiva, como la capacidad de registrar y elaborar diferencias sensoriales. Esta definición, muy distinta a la del afecto, entre otras cosas está unida a la noción de «bit», es decir, a la más diminuta diferencia distinguible, fundamental en la teoría de la información. De ahí que se una a la cibernética en general, incluido el lenguaje digital de los ordenadores. Ésta se halla en completa consonancia además con las teorías del matemático y epistemólogo de las ciencias inglesas Spencer-Brown, quien postula que todo el mundo cognoscitivo puede reconstruirse mediante el establecimiento de una sucesión de diferencias y de diferencias de diferencias. Bajo este ángulo, incluso los animales primitivos ya disponen de sencillas capacidades cognoscitivas.

Con el término de lógica (en sentido amplio) hay que incluir en el contexto de la lógica afectiva el modo en que los diferentes elementos cognoscitivos son seleccionados y unidos entre ellos para formar entidades cognoscitivas («construcciones del pensamiento») más amplias. Esta definición intencionadamente extensa implica la existencia de varias formas de lógica, una noción adoptada igualmente por la filosofía y la teoría de la ciencia moderna y, en particular, por el constructivismo moderno. Un constructivismo no radical pero sí relativo es igual- 
mente afrontado por la lógica afectiva por razones que no pueden detallarse en este momento.

Mi segunda tesis postula que los afectos no sólo acompañan al pensamiento y al comportamiento sino que también en buena medida los guían y los organizan.

En ese sentido los afectos no funcionan únicamente como un proveedor de energía, es decir, como motor (y también a veces como freno, como por ejemplo en los estados depresivos) de cualquier actividad intelectual, como creía Piaget. Más allá influyen continuamente en el pensamiento y la acción mediante una serie de «efectos conmutadores» o de «efectos operadores generales y específicos» siguiendo mi terminología (un operador es una variable que influye en otra variable, modificándola). Los efectos operadores generales de los afectos son, en su origen, idénticos en todo estado afectivo. Así, cualquier estado afectivo influye de continuo, en primer lugar, en el foco de atención. Al mismo tiempo, determina en todo momento lo que es importante o lo que no. La atención se centra preferentemente en percepciones o pensamientos que se corresponden con el estado afectivo ordinario, ignorando lo que no le incumbe.

En un humor ansioso o depresivo, por ejemplo, se acentúan sobre todo los aspectos peligrosos u oprimentes de una sola e inmutable realidad que le rodea.

En un humor alegre o distendido, por el contrario, percibimos en general sus aspectos agradables. La memoria igualmente depende del estado afectivo ordinario.

En un estado eufórico, ante todo se tiende a registrar y a poner en movimiento recuerdos placenteros, mientras que los recuerdos desagradables surgen a la superficie en un estado depresivo. El humor afectivo condiciona además la selección de elementos cognoscitivos que a continuación se ensamblarán en construcciones cognoscitivas más extensas, es decir, en el tipo de lógica del que hemos hablado aquí. Un ejemplo: cuando surge un problema conyugal, pensamos más en todo el mal que nuestra pareja nos ha hecho en el pasado que en el que nos hace en el presente y en el que nos hará en el futuro. Con todos estos elementos nos construimos una cadena de argumentos agresivos propios de una lógica lacerante. En otras palabras, nuestro razonamiento sigue una «lógica de la cólera», si podemos definir así este modo de pensar sin que violemos inevitablemente las leyes de la lógica formal. En el marco de una «lógica del amor»y, de manera singular, en el estado amoroso, percibimos, el mundo de otra manera. Construimos una «realidad» diferente. Y así igualmente existe la lógica del miedo, la lógica de la alegría, la lógica del duelo, etc.

Estos efectos operadores generales, semejantes en todos los afectos, merecen distinguirse de los efectos específicos de los afectos que influyen en el pensamiento y en la acción de diferente modo según sea un afecto u otro. El interés y la 
curiosidad, por ejemplo, activan las energías emocionales y las dirigen hacia determinados objetos. El miedo se distingue porque provoca un distanciamiento, y, eventualmente, la huida ante objetos que creemos peligrosos. Los sentimientos agresivos como la rabia o la cólera establecen a su vez, en primer lugar, unos límites («hasta aquí hemos llegado»), que nos alejan de todo. Desde un punto de vista evolutivo la agresividad sirve, sobre todo -como así lo ha demostrado Konrad Lorenz- para defensa del territorio o, si somos más precisos, para defender su propia identidad. Sentimientos agradables como la alegría, el placer o el amor engendran, por el contrario, la proximidad y el cariño que, a su vez, estará seguido por el desapego y la tristeza (por el «trabajo de duelo», según Freud) en caso de pérdida del objeto amoroso. Ahora bien, según la investigación etológica comparativa, todos esos sentimientos y comportamientos de base -la curiosidad, la huida o la defensa del territorio, el arraigo o el desapego- son de vital importancia desde el punto de vista evolutivo. En otras palabras, no poder sentir interés, miedo, rabia, placer, alegría y no disponer de ningún medio para reponerse de una pérdida, supuesta la incapacidad de «hacer duelo», equivaldría a un peligro mortal. Darwin ya en su momento se sentía profundamente interesado por la función de los sentimientos en los animales y en los seres humanos, aunque este aspecto de su trabajo sea mucho menos conocido que sus descubrimientos seculares sobre la evolución de las especies.

Los afectos son por lo tanto -aunque conviene precisarlo- fenómenos claramente sensatos («sistemáticamente razonables», como diría Niklas Luhmann) ${ }^{7}$. Por el contrario, lo que parece totalmente «irracional» es precisamente el «pensamiento sin sentimientos», según una fórmula del conocido terapeuta sistémico Fritz B. Simon. La evaluación afectiva subdivide la realidad vivida en categorías vitales tales como «peligroso/no peligroso», «agradable/desagradable», etc. Operadores afectivos hondamente inconscientes se encargan, partiendo de esta base, de la adaptación autorreguladora de la atención, la memoria, el pensamiento y el comportamiento. En suma, los afectos se corresponden -para utilizar un concepto fundamental en informática- con reductores de complejidad enormemente eficaces, indispensables para comprender el mundo y la realidad cotidiana que nos circunda.

Evidentemente, podríamos alegar que todo esto está muy bien, aunque solo podría aplicarse a estados con gran carga emocional y no al pensamiento cotidiano de poca carga emotiva. Pero examinemos este pensamiento cotidiano más de cerca. Éste incluye todo lo que es nuevo y excitante, pero que ha terminado por convertirse en algo banal a fuerza de repetirlo. Habitualmente las emociones 
intensas del principio devienen inconscientes poco a poco. No obstante, éstas conservan, en su nivel de inconsciencia, la mayor parte de sus efectos sobre el pensamiento y el comportamiento. Por ejemplo, sólo gracias a los efectos subyacentes de las intensas alegrías y angustias vividas en fase de aprendizaje somos capaces de conducir un coche sin sufrir un accidente. Frenamos automáticamente con prudencia ante una curva y aceleramos tranquilamente cuando la vía está libre. Al mismo tiempo, seguimos desconfiando de las carreteras secundarias donde perros, niños, etc. podrían cruzar de repente. Sin saber muy bien cómo lo hemos conseguido, nos encontramos de pronto al final de nuestro viaje. Sin embargo, todas las emociones que son conscientes en su origen surgirán inmediatamente a la superficie cuando se produzca algo excepcional. La multitud de pensamientos y de costumbres en apariencia exentas de afectos que acompañan nuestra vida cotidiana obedecen a reglas semejantes. Una ingente cantidad de sutiles regulaciones afectivas inconscientes están de continuo trabajando en todas nuestras maneras de pensar, nuestras «mentalidades», ideologías o prejuicios (que normalmente atribuimos a los demás) que parecen «triviales».

Examinado bajo el ángulo de la economía energética, el sentido de dicho mecanismo está muy claro. Se trata del rodaje progresivo de nuestras maneras de pensar, aprendidas primeramente a cambio de inversiones emocionales considerables, de manera que terminen por funcionar con un desgaste enérgico mínimo -por así decirlo- con servoreguladores emocionales. «Pensar es actuar a modo de prueba con un pequeño desgaste de energía», decía Freud ya en un contexto similar. A la inversa, se podría comprender también la acción -en forma de experiencia científica-, como un pensamiento a prueba, acompañado por un gran desgaste energético.

Cabe destacar que todo esto sirve igualmente, visto de cerca, para el pensamiento abstracto y matemático. Pues las contradicciones e incongruencias abstractas son tan penosas y desagradables (es decir, que consumen emocionalmente), como las contradicciones concretas. Las soluciones justas, por el contrario, son agradables, pues, reducen la tensión por su economía de energía emocional. ¡No por casualidad el matemático se regocija cada vez que halla una solución particularmente «elegante» de cierto problema!

La lógica afectiva postula, por lo tanto, que la búsqueda de este «pensamiento placentero», de esta economía de energía emocional, domina y dirige el pensamiento abstracto tanto como la acción concreta. Como ocurre en un determinado paisaje, los caminos de pensamiento viables, caracterizados por una reducción del gasto energético, en primer lugar, deben ser encontradas y probadas, también en los «paisajes abstractos», con la ayuda de energías emocionales considerables, antes de que puedan convertirse en banalidades de las que nos servimos sin esfuerzo. 
Innumerables investigaciones científicas ilustran esta dinámica afecto-cognoscitiva siempre semejante, desde el famoso «eureka» de Arquímedes con su descubrimiento del principio sobre el peso específico, pasando por la revolución copernicana que nos condujo de una noción geocéntrica a una comprensión heliocéntrica de nuestro mundo hasta el desciframiento de la estructura del genoma en la doble hélice por Crick y Watson. El descubrimiento de la novedad emerge, por decirlo así, de la tensión emocional dolorosa, de la que una especie de sufrimiento corresponde sin duda, en última instancia, a la angustia. Volveremos a esta idea de base ya enunciada por Freud así como por Heidegger.

Mi tercera tesis afirma que los sentimientos, pensamientos y comportamientos vividos simultáneamente, en una situación determinada, tienden a agravarse en la memoria en forma de unidades funcionales.

Estas unidades se reactualizarán en situaciones semejantes y se diferenciarán y se modificarán eventualmente bajo la influencia de nuevas experiencias. Se corresponden, por lo tanto, con verdaderos «programas integrados de sentimiento, pensamiento y comportamiento» (que llamaremos «programa SPC»). De estos programas se constituyen los elementos de construcción fundamentales para el «aparato psíquico» (Freud). Puede entenderse la psique como un entrelazado complejo de programas SPC. Esto puede ilustrarse mediante un simple ejemplo: el proverbio «Niño quemado no se arrima al fuego» generaliza la observación de que la percepción del fuego, la experiencia del dolor y el terror sentidos por un inocente niño que, por ejemplo, tendió por curiosidad su dedo cerca de una llama, instantáneamente se transformaron en reflejo de un desvío condicionado, es decir, en un «programa SPC» integrado que protegerá al niño para siempre en la repetición de la misma experiencia dolorosa. Este simple «programa» unido al fuego, estará después progresivamente diferenciado y parcialmente modificado mediante nuevas experiencias. Algo análogo ocurrirá ya ante cualquier otro tipo de experiencia, sean otras personas, otros lugares, objetos o actividades, o incluso ante nociones abstractas como son por ejemplo las teorías o las ideologías. Sabemos perfectamente que los programas SPC adquiridos en la primera infancia pueden ser el origen de líneas de comportamiento que persisten durante toda la vida. Esto ocurre generalmente con formas de comportamiento que los psicoanalistas llaman reacciones de transferencia.

Dependiendo de los efectos operativos de los sentimientos sobre el pensamiento y el comportamiento que hemos descrito, los programas SPC teñidos por cierta coloración afectiva tenderán a aglomerarse y a formar, poco a poco, lo que podríamos llamar «rieles afectivo-cognoscitivos». Incluso los fenómenos transferenciales en sentido psiconalítico nos proporcionan excelentes ilustraciones. Como consecuencia de una relación infantil traumática con un padre excesivamente severo, por ejemplo, puede desarrollarse -y progresivamente transformarse 
en un rasgo de la personalidad- una actitud excesivamente ansiosa, servil y sumisa en apariencia pero al mismo tiempo agresiva y sublevada en el fondo hacia cualquier persona de autoridad masculina. Mediante la combinación de distintos «rieles», verdaderos «mundos afecto-cognoscitivos propios» pueden irse formando progresivamente. Así se desarrollará acaso a lo largo del tiempo, excepcionalmente, la convicción inquebrantable de que no se puede confiar en ninguna persona de rango social superior y, al final, en ninguna persona sin más. Las construcciones afectivo-cognoscitivas del mismo género, específicas para cada persona, para cada grupo o para una cultura determinada están en la base de todo tipo de ideologías marginales como por ejemplo las visiones del mundo de tipo sectario, fundamentalista, «autista» o delirante. Veremos, a continuación, más de cerca las consecuencias nefastas a las que semejantes «rieles»o «mundos propios» nos pueden a veces inducir.

Mi cuarta tesis afirma, de una manera que puede parecer un poco enigmática a primera vista, que el grande está dentro del pequeño y el pequeño está dentro del grande, o incluso, que la psique posee una estructura fractal.

La noción de fractalidad proviene de las teorías del caos y de la complejidad. Puede traducirse por autosemejanza en cualquier dimensión, pequeña o grande, y significa que ciertas estructuras obedecen, en todos los tamaños posibles, a los mismos principios de construcción. Esas autosemejanzas que llamamos «fractales» se han detectado en un gran número de fenómenos naturales y culturales, en la estructura de las nubes tormentosas llamadas «nubes a modo de coliflor», en las que la forma de una nubecilla se asemeja a la forma de toda la nube, a través de innumerables estructuras geológicas, botánicas o animales y llegando hasta estructuras urbanísticas y en parte a los aspectos de la dinámica socioeconómica.

Ahora bien, parece muy verosímil, desde el punto de vista de la lógica afectiva, que la variedad infinita de fenómenos psicodinámicos y sociodinámicos obedece igualmente a un principio de construcción de tipo fractal, acaso porque el principio de la aplicación de una única regla de formación (de un algoritmo) capaz de engendrar una gran riqueza de formas es particularmente económica. Ahora bien, acabamos de ver que dicha regla se pone en marcha bajo la forma de un efecto operatorio sobre pensamiento y acción, descritos más arriba.

Las emociones individuales y colectivas estimulan, focalizan y organizan, de hecho, el pensamiento y el comportamiento a todos los niveles fundamentalmente por igual. Así no sólo las personas sino también todas las naciones en su totalidad pueden quedarse literalmente hipnotizadas durante semanas por los campeonatos mundiales de fútbol o en duelo por un acontecimiento como la muerte de lady Diana. O lo que es mucho más grave, el pensamiento colectivo puede verse aprisionado, como cualquier pensamiento individual, por la lógica de la rabia, del miedo o de la desesperación que puede engendrar la guerra. En el marco del con- 
flicto palestino-israelí, por ejemplo, los efectos operadores de esos sentimientos sobre el pensamiento y la acción se manifiestan de hora en hora como de año en año, y de decenio en decenio de manera semejante a todos los niveles posibles, individuales, familiares y nacionales.

Incluso dentro del nivel individual podemos encontrar innumerables variaciones de un solo y único principio de formación. Lo que llamamos «estructura de la personalidad», por ejemplo, se manifiesta a menudo de manera semejante en minúsculos fragmentos del comportamiento a corto plazo -así, en un gesto momentáneo- al igual que en una compleja reacción de transferencia a largo plazo. Este fenómeno, por otro lado, ha sido explotado por tests de personalidad como los test de Rorschach, donde los rasgos de la personalidad se detectan a través de actos de percepción aislados, estimulados por manchas de tinta que tienen formas equívocas. Como con el fenómeno del «estilo» en el arte, la fractalidad de la estructura de la personalidad puede describirse mediante la fórmula «lo más grande dentro de lo más pequeño, lo más pequeño dentro de lo más grande». Es esta estructura fractal la que nos permite reconocer a un artista en cada resquicio de su obra, una vez que ésta nos es conocida.

Otro aspecto particularmente interesante de la fractalidad de la psique consiste en el hecho de que en toda emoción, el resto de las emociones (de base) en cierta medida se ven siempre incluidas: encontramos una pizca de amor en el odio, un asomo de miedo en la rabia, un atisbo de tristeza en la alegría y viceversa. Mucho antes del psicoanálisis este hecho había sido ya descrito por numerosos poetas y pensadores. De manera metafórica podría representarse por pequeños toques de color contrastados en una superficie de apariencia monocroma o, mejor aún, por minúsculas rugosidades escondidas en una pared rocosa que pareciese, a primera vista, lisa como un espejo.

Estos elementos alternativos, ocultos en un estado de sufrimiento en apariencia uniforme como por ejemplo en una depresión grave, pueden ser explotados en la psicoterapia. El psicoterapeuta puede, por así decirlo, buscarlos con lupa, luego focalizarlos de manera selectiva y, finalmente, ampliarlos sistemáticamente de manera que se vaya descubriendo poco a poco una vía de escape.

Mi quinta tesis es, en cierto modo, el resumen de las cuatro anteriores. Ésta afirma que los afectos son los motores y organizadores esenciales de toda evolución psíquica y social.

Si indagamos entre las energías que ponen en marcha la dinámica de los procesos psicosociales, sea cual sea su tamaño, topamos siempre con la fuerza de las emociones, tras considerar todos los hechos biográficos, socioeconómicos o religiosos. Pretendo decir que nadie puede comprender adecuadamente los conflictos sociales, las erupciones de violencia, las revoluciones y los movimientos políticos $-\mathrm{y}$, realmente, cualquier dinámica psicosocial- a través de aspectos cognoscitivos 
si no tiene en cuenta de manera sistemática las interacciones entre cognición y emoción. Precisamente eso es lo que ha sido ignorado durante mucho tiempo por la mayor parte de las ciencias humanas, a excepción, sin embargo, del psicoanálisis. En toda la obra del gran sociólogo y revisador de las teorías sociales modernas Niklas Luhmann, por ejemplo, no encontramos prácticamente ninguna huella de semejante idea, a pesar de que haya escrito libros completos sobre la confianza y el amor. Incluso cuando no muestra interés exclusivamente por sus variaciones semánticas a través de las culturas (como en el amor), los sentimientos resultan ser para él una especie de signo de alarma perturbadora proveniente del ámbito individual. Como principio, por lo tanto, no tendrían cabida en la sociología. Es significativo, sin embargo, que igualmente en los «sistemas de consciencia individuales», como así llama al campo de los fenómenos psíquicos, las emociones solo ocupen uno de los lugares más marginales ${ }^{8}$.

$\mathrm{Si}$, por el contrario, entendemos los estados emocionales como energías omnipresentes, o si tendemos a destacar el hecho de que los sentimientos son altamente contagiosos, sobre todo si emanan de personalidades carismáticas como gurús u otros «individuos alpha», resulta muy claro que es de allí, y no de ningún otro sitio, de donde provienen las fuerzas fundamentales que azuzan y mueven la dinámica psíquica y social. Energías emocionales individuales y dirigidas en un mismo sentido pueden converger hacia flujos colectivos extremadamente poderosos, dando así lugar al surgimiento de fenómenos macrosociales totalmente nuevos, como por ejemplo la aparición de nuevas formas de organización y diferenciación colectiva, movimientos sociales, modas, etc. A través de todos estos niveles, observamos, de hecho, los mismos efectos movilizadores y estructurantes de los sentimientos sobre el pensamiento y la acción que hemos descrito más arriba. La histeria o el pánico de las masas, la formación de sectas, los extremismos nacionalistas u otros proporcionan ejemplos sorprendentes. Igualmente en el plano colectivo existen «mundos afectivo-cognoscitivos» o «modos de pensar» que están dominados por la rabia o el odio, así como existen mundos dominados por el amor y la confianza. Su lógica se ve organizada y estructurada consecuentemente. En ningún otro lugar estos «mundos afectivo-cognoscitivos» específicos son tan evidentes como en las famosas «espirales de violencia» tal y como por ejemplo están a punto de resurgir a una velocidad inquietante en el conflicto palestino-israelí, en Macedonia, o en numerosos puntos neurálgicos del mundo.

Otro fenómeno que no puede ser adecuadamente comprendido más que por una aproximación simultánea energética y caos-teórica de los afectos son los saltos inesperados que surgen con una frecuencia notable en todo tipo de procesos 
psíquicos y sociales. Desde hace más de veinte años, la teoría del caos estudia sistemáticamente las condiciones de aparición de este tipo de «bifurcaciones» en los sistemas dinámicos extremadamente variados. Ésta ha podido demostrar que repentinos saltos no lineales hacia otras modalidades globales de funcionamiento se producen de manera apremiante cada vez que la energía introducida en un sistema dinámico no puede disiparse por los conductos habituales. La máquina a vapor explota cuando la presión aumenta hasta el punto que no puede liberarse por las válvulas previstas. Numerosos ejemplos demuestran un fenómeno semejante cuando la tensión emocional aumenta de manera desmesurada en un sistema psíquico o social. Una paz frágil de repente se convierte en guerra abierta (y por lo tanto en otro modo de funcionamiento energético global) cuando la tensión política alcanza un punto crítico. Algo semejante se produce en el ámbito microsocial cuando, tras una fase de invectivas verbales, dos protagonistas llegan a las manos. La búsqueda sistemática de los efectos de lo que llamamos «emociones expresadas»-expressed emotions $-^{-9}$ ha demostrado que la bifurcación que conduce de un comportamiento normal a un comportamiento psicótico obedece a mecanismos similares. Lo mismo sirve para innumerables crisis conyugales, profesionales, bursátiles o políticas.

No es de extrañar, por otro lado, que igualmente observemos en este tipo de procesos «efectos mariposa» típicos -fenómeno no lineal que las ciencias del caos han explorado de manera sistemática-. Se trata de cómo, en ciertas condiciones, minúsculas causas pueden engendrar enormes efectos. Es también lo que se ha descrito como la imagen de la gota proverbial que desborda el vaso o de la chispa que hace explotar el barril de pólvora.

Un famoso ejemplo histórico fue el atentado de Sarajevo que desencadenó la primera guerra mundial en 1914. No menos espectacular fue el efecto de la apertura de una pequeña brecha en el muro de Berlín en 1989 que condujo, en fin de cuentas, a la caída de todo el inmenso imperio soviético.

Antes de abordar las consecuencias prácticas y generales de esta nueva manera de comprender los procesos psicosociales conviene decir algunas palabras sobre las relaciones entre la lógica afectiva y los tres accesos al comportamiento humano, en mi opinión, más importantes de los que disponemos hasta este momento: el acceso psicodinámico, el sociodinámico y el neurobiológico. La lógica afectiva no está en contradicción con ninguno de ellos, sino todo lo contrario: los une y los ilumina mutuamente. En este sentido se correspondería menos con una nueva teoría que perturbase al resto, y más con una teoría de base, o metateoría que ordenase e integrase sus aspectos aparentemente heterogéneos dentro de 
una perspectiva superior, reduciendo su complejidad. Se vienen persiguiendo metas parcialmente semejantes en la sociedad de neuropsicoanálisis recientemente fundada en Londres así como por la «psicoterapia general» desarrollada desde hace algunos años por Klaus Grawe ${ }^{10}$.

Sus efectos emocionales explican las fuerzas básicas, de orden biológico en última instancia, que dinamizan, estructuran e integran los sistemas psíquicos o sociales auto-organizadores. En cualquier tipo de dinámica psicosocial resultan ineludibles las profundas influencias afectivas. Merecen, por lo tanto, un lugar central y no sólo marginal en nuestra manera de comprender a la gente y al mundo. Según la lógica afectiva, por ejemplo, los mecanismos de represión, de anclaje y de transferencia (en sentido psicoanalítico), se corresponden solo con casos especiales de efectos de selección y represión que en general todos los afectos ejercen sobre el pensamiento y la memoria. Los fenómenos del consciente y del inconsciente se presentan igualmente bajo un nuevo prisma. La atención consciente se presenta como un modo de funcionamiento particularmente oneroso desde el punto de vista energético y que queda reservado esencialmente a tareas nuevas o excepcionalmente difíciles, así como a aspectos potencialmente peligrosos o particularmente interesantes. El inconsciente representa, por el contrario, el modo de funcionamiento más económico posible. Por lo tanto, constituye el objetivo final de cualquier aprendizaje, y, en última instancia, de cualquier aprendizaje en psicoterapia. Pensemos una sola vez más en el ejemplo de la conducta automovilística. No tiene, pues, únicamente sentido el famoso lema freudiano «Lo que era Ello habrá de ser Yo», sino también su contrario: «Lo que era Yo habrá de ser Ello».

\section{Consecuencias prácticas y generales}

Si omnipresentes energías afectivas ejercen realmente todos los efectos de movilización, organización y reducción de complejidad sobre el pensamiento individual y colectivo postulados por la teoría de la lógica afectiva, ella tendrá forzosamente múltiples consecuencias en los ámbitos más variados de la vida familiar y profesional del día a día a través del comercio, la publicidad y la política, pasando por la pedagogía, la psicoterapia e, incluso, la teoría del conocimiento y de la filosofía. En este punto no podré abordar más que algunos aspectos de interés general.

En todas partes, donde la comunicación es importante - ¿y dónde no lo sería?- hay que tener en cuenta los continuos efectos emocionales sobre el pensa- 
miento y el comportamiento. Pues la comunicación está siempre afectivamente coloreada, incluso cuando se presenta como «neutra» $\mathrm{u}$ «objetiva» (como ya he dicho, esas disposiciones igualmente se corresponden con estados afectivos específicos). Para que alcance un «tono de fondo» relajado o distendido, jovial o irritado en una comunicación, importa casi tanto como su contenido y, en ocasiones, incluso mucho más. Cuando el color emocional de un mensaje se corresponde con el humor afectivo del receptor, el mensaje será mucho más fácilmente comprensible y aceptado que en el caso contrario. Por esta razón cualquier vendedor, político, pedagogo o psicoterapeuta experimentado se esfuerza, primeramente, en crear un ambiente emocional propicio antes de «entrar realmente en materia». O mejor dicho: comienza por entrar él mismo en el mundo afectivo propio de su interlocutor o de su cliente; conscientemente se deja «infectar» en cierta medida -este es exactamente el sentido de la atención sistemática llevada a la transferencia o a la contratransferencia en psicoanálisis- $\mathrm{y}$ «transmite» a continuación su mensaje sobre una «extensa onda» emocionalmente similar. Y por así decirlo se conecta a los «programas integrados del sentir, del pensar y del comportarse» que detecta en su interlocutor; luego los refuerza por un lado y los mitiga por otro; introduce en ellos nuevas informaciones (notemos de paso que cualquier información posee una connotación afectiva, sin la cual no prestaría atención alguna), consiguiendo provocar poco a poco cambios efectivos.

Si además tenemos en cuenta el hecho de que estos afectos, tal y como acabamos de definirlos, se manifiestan no solo en «nuestra cabeza» sino también (y muy a menudo en primer lugar) en el cuerpo, nos daremos cuenta de que no solo la lengua hablada sino también que la acción común (por ejemplo el deporte, la danza, el paseo, el trabajo manual) pueden constituir excelentes soportes para una comunicación terapéutica eficaz. No por casualidad los hombres de Estado particularmente perspicaces se pasean a veces por el bosque o frecuentan incluso la sauna para favorecer el desbloqueo en situaciones delicadas. Un gran número de métodos psicoterapéuticos, que no se basan únicamente en la palabra sino también en la vivencia personal, exploran precisamente este tipo de mecanismo.

De igual manera, tiene una gran importancia el ambiente emocional del medio donde se desarrolla cierta comunicación terapéutica o de otra índole. Así por ejemplo ha podido comprobarse de manera particularmente llamativa en el marco de un proyecto piloto que pusimos en marcha hace dieciocho años con el nombre de «Soteria Berna». En lugar de calmar a los pacientes psicóticos agudos mediante dosis elevadas de medicamentos, nosotros les tratamos en primer lugar y con gran éxito con la compañía humana constante en un medio terapéutico específicamente concebido para disminuir la angustia y la tensión. Esta experiencia, impulsada por investigaciones comparativas crecientes, confirma la hipótesis mencionada más arriba según la cual la aparición de turbaciones psicóticas está estrechamente relacio- 
nada con el nivel de tensión emocional que se desarrolla en una persona vulnerable. Semejantes correlaciones se han ido detectando en otro tipo de afecciones psíquicas. El lector interesado encontrará precisiones sobre el tema en el libro recientemente publicado en alemán: ¿Cómo actúa Soteria? Una evaluación crítica de un tratamiento atípico de la psicosis ${ }^{11}$.

Otras consecuencias prácticas de la lógica afectiva merecen también ser tratadas como, por ejemplo, sus aplicaciones pedagógicas o profesionales, o sus implicaciones en el manejo de las relaciones humanas en el plano institucional ${ }^{12}$. Tratemos todavía algunos aspectos generales. Desde siempre los pensadores y naturalmente los poetas han estado muy interesados por los sentimientos. Entre los primeros no mencionaré más que a Platón, Spinoza o más próximo a nosotros, Heidegger, quien vincula en un último análisis cualquier tipo de actividad intelectual a la angustia, en una palabra ${ }^{13}$. Su discípulo y crítico Bollnow ha ampliado esta aproximación hasta una antropología general de los afectos que coincide de manera chocante con las tesis centrales de la lógica afectiva ${ }^{14}$. De cualquier manera, la noción hacia la que todas estas contribuciones convergen -y convergen hacia la conciencia de que un pensamiento libre de afecto no existe ni puede existir- no nos dirige sólo a una comprensión más global sino también más realista, más humana y quizá más grata del hombre y del mundo. Y nos induce, igualmente, a hacer una revisión dolorosa de nuestra comprensión, siempre demasiado idealista, de nosotros mismos. Los dramas privados y colectivos que vivimos a diario -no menciono, aparte del terror cotidiano de la pequeña pantalla, más que algunas claves de los últimos diez años como la guerra del Golfo, la de Ruanda, Bosnia y Kosovo, Israel y Palestina, sin hablar del Holocausto y de otros horrores de un pasado no tan lejano- no son azarosos sino que están profundamente enraizados en nuestras disposiciones innatas al sentir, pensar y actuar. Las energías emocionales, semejantes al fuego, al viento o al agua, corresponden a fuerzas elementales de la naturaleza. Desencadenadas, pueden engendrar terribles consecuencias. Sublimadas, por el contrario, son capaces de dar lugar a fenómenos extremadamente diferenciados.

En consecuencia, siempre puede haber nuevas atrocidades, si las condiciones son propicias, casi cien años más tarde nada queda, ¡ay!, de la esperanza, antaño formulada por Sigmund Freud y más tarde también por Norbert Elias, acerca de la domesticación cultural de las emociones. El dolor, el miedo y la violencia no se han sublimado (en una época de bombas de hidrógeno, de

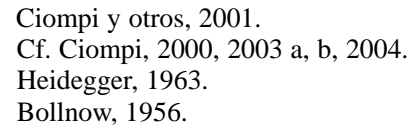


napalm, de bombardeos y misiles de precisión), sólo se han vuelto anónimos, han sido racionalizados y convertidos, de manera cada vez más refinada, mediante procedimientos tecnológicos. La sexualidad desenfrenada nada tiene que ver con el amor sublime y mucho menos la pornografía; ni tampoco la perversión tecnológica progresiva de procesos vitales como la concepción y el embarazo, el nacimiento y la muerte que antes eran sagradas. ¿Dónde queda la consciencia de que cada ser humano es una parte (un fragmento o un «fractal») de un gran ensamblaje, un sensor, así como -en una medida infinitesimal que sea- un actor y co-autor responsable en el inmenso lienzo de las causas y efectos que constituye la «realidad»? Después de las últimas noticias de horror, como las de los Estados Unidos, no cuentan en serio ya la modificación global del clima, ni que el parlamento brasileño permita la deforestación de la mitad de la Amazonía, ni que pronto no habrá elefantes o grandes monos antropoides, ni que la dignidad individual y el genoma humano no serán en adelante nada sagrados, tras lo cual no se esconde sólo (como se pretende a menudo) el frío calculo del pensamiento racional. Desde hace mucho tiempo la industria farmacéutica, el genio genético, la ciencia no promueven la investigación por el bien de la humanidad en primer lugar, y mucho menos por amor al puro saber sino sólo por su propio provecho monetario y estadístico. Y tras la lógica del provecho a toda costa se perfilan muy bien, de manera disimulada siempre, las mismas emociones de fondo que existen desde siempre, desde el origen del pensamiento y de la acción del hombre: el miedo, el terror, la codicia y la envidia, la carrera hacia la felicidad, el poder y la posesión, así como también, sin duda, y extrañamente entremezclada con todo esto, una amagalma de «sentimientos positivos».

Porque, evidentemente, también existe el reverso de la moneda: mezcladas y dosificadas de manera diferente encontramos, de hecho, las mismas energías emocionales que las que estimulan el camino hacia el provecho en las transformaciones culturales, artísticas o espirituales más grandes del hombre, en su eterna búsqueda de belleza y equidad, en su nostalgia de la armonía y la paz, en su capacidad de cuidar, cultivar y amar.

En fin de cuentas, ¿qué es el hombre?, o mejor dicho, siguiendo la perspectiva de la lógica afectiva, ¿en qué se está convirtiendo? ¿Es un animal muy terrible, pues, siendo terriblemente inteligente, es el más atroz de todos los depredadores o, por el contrario, ¿es un milagroso venero espiritual, una verdadera «corona de la creación»? Lo ignoramos, aunque de momento no presentimos nada bueno. Sólo sabemos que tiene y tendrá siempre la última palabra. Ahora y siempre nuestra suerte estará echada durante el transcurso de esta lenta y desmesurada evolución, magnífica y cruel a la vez, que ya ha inventado centenares de millones de formas de vida y que ha rechazado otras tantas. 
Por sorpresa, desde las ciencias de la evolución -y precisamente con esta nota moderadamente optimista deseo terminar mi reflexión sobre las relaciones entre pensamiento y sentimiento- nos llega desde hace poco un nueva luz de esperanza. Según sus últimas investigaciones ya no es el brutal poder del más fuerte el que conduce a las mejores posibilidades de sobrevivir. Es más bien la capacidad de trabajar en armonía, de colaborar en una forma diferente. Por lo tanto, es una forma de economía, de armonía y en última instancia, de amor, la que se revela como la invención más fructífera de la evolución a largo plazo. Y precisamente así podría -o incluso debería, desde la perspectiva de la «racionalidad sistémica»lograrse acaso, finalmente, nuestra salvación.

\section{BIBLIOGRAFÍA}

(1) Bollnow, O. F., Das Wesen der Stimmungen, Fráncfort del Meno, Klostermann, 1956.

(2) CiomPI, L., Affektlogik. Über die Struktur der Psyche und ihre Entwicklung. Ein Beitrag zur Schizophrenieforschung, Stuttgart, Klett-Cotta, 1982. The Psyche and Schizophrenia. The Bond between Affect and Logic, Cambridge (Mass.) y Londres, Harvard University Press, 1988.

(3) CiompI, L., Die emotionalen Grundlagen des Denkens. Entwurf einer fraktalen Affektlogik, Gotinga, Vandenhoeck y Ruprecht, 1997.

(4) CiompI, L., Gefühle, Affekte, Affektlogik. Ihr Stellenwert in unserem Menschen und Weltverständnis, Viena, Picus, 2002.

(5) CıOMPI, L., «Affektlogik, affecktive Kommunikation und Pädagogik. Eine wissenschaftliche Neuorientierung», en UNTERWEGER, E., ZIMPRICH, V. (eds.), «Neuorientierung», en UNTERWEGER, E.; Zimprich, V. (eds.), Braucht die Schule eine Psychotherapie?, Viena, Orac., 2000, pp. 3-17.

(6) Freud, S., Beiträge zur Psychologie des Liebeslebens. Gesammelte Werke, VIII, Londres, Imago Publishing, 1940, p. 91 (y Über Sublimation).

(7) Damasio, A., Descartes Error. Emotion, Reason and the Human Brain, Nueva York, Grosset/Putnam, 1994.

(8) Freud, S., Neue Folge der Vorlesungen zur Einführung in die Psychoanalyse, Gesanmmelte Werke, XV, Londres, Imago Publishing, 1940, p. 96.

(9) Freud, S., «Beiträge zur Psychologie des Liebeslebens», Gesammelte Werke, VIII, Londres, Imago Publishing, 1940, p. 91 (y Über Sublimation), GolEMAN, D., Emotional Intelligence, Why it can Matter More than IQ, Nueva York, Bantam, 1995 (Emotionale Intelligenz, Múnich y Viena, Hanser, 1996).

(10) Grawe, K., «Grundriss einer allgemeinen Psychotherapie», Psychotherapeut, 40, 1995, pp. $130-145$.

(11) Hatfield, E.; Caciopro, J. T.; Rapson, R. L., Emotional Contagion, París, Cambridge University Press, 1994.

(12) Heidegger, M., Sein und Zeit, Halle del Saale, 1927.

(13) Hesse, H., Die Nürnberger Reise, Berlín, 1927, p. 43.

(14) KernBerg, O., «New Perspectives in Psychanalytic Affect Theory», en Kellermann, H.;

PlutchiK, R., Emotion Theory, Research and Experience, Nueva York, 1990, pp. 115-131. Bollnow, O. F., Das Wesen der Stimmungen, Fráncfort del Meno, Klostermann, 1956. 
(15) Kleinginna, P. R.; Kleinginna, A., «Cognition and Affect. A Reply to Latarus and Zajonc», Am. Psychologist, 40, 1985, pp. 470-471; CiomPI, 1997, cap. 1; LuHMAnN, N., «Die Autopoiesis des Bewusstseins», Soziale Welt, 36, 1985, pp. 402-446.

(16) LeFF, J.; VAughn, C.; Expressed Emotions in Families. Its significance for Mental Illness, Nueva York, Londres, Guilford Press, 1985; Luhmann, N., Die Gesellschaft der Gesellschaft, Suhrkamp, Fráncfort del Meno, 1997.

* Wiener Vorlesung (9-V-2001), publicado bajo el título Gefühle, Affekte, Affektlogik. Ihr Stellenwert in unserem Menschen und Weltverständnis, Viena, Picus (cf. Ciompi, 2002). Está traducido de la versión del autor, julio 2004.

** Profesor emérito Luc Ciompi. Director de formación en Psiquiatría Social del Hospital Clínico Universitario, Facultad de Medicina, Universidad de Berna (Suiza). 